\begin{tabular}{|c|c|}
\hline $\begin{array}{l}\text { Conceptos clave para la formaci } \\
\text { del Diseñador Industrial }\end{array}$ & $\begin{array}{l}\text { Isaac Acosta Fuentes (coordinador) } \\
\text { Marcio Dupont Caballero de Carranza } \\
\text { Brenda García Parra } \\
\text { Sandra Luz Molina Mata } \\
\text { Sustentabilidad y diseño industrial } \\
\text { p. [209]-230 } \\
\text { En: } \\
\text { Gutiérrez-Ruiz, Francisco Javier (coordinador). } \\
\text { Conceptos clave para la formación del Diseñador } \\
\text { Industrial. Ciudad de México: Universidad Autónoma } \\
\text { Metropolitana, Unidad Azcapotzalco, } 2012.312 \text { páginas. } \\
\text { Fuente: ISBN 978-607-477-626-3 } \\
\text { Relación: } \\
\text { http://hdl.handle.net/11191/7663 } \\
\text { https://doi.org/10.24275/uama.352.7663 }\end{array}$ \\
\hline
\end{tabular}

\begin{tabular}{|c|c|c|}
\hline 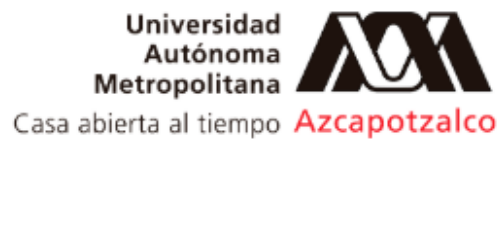 & $\begin{array}{l}\text { División de Ciencias y Artes para } \\
\text { el Diseño }\end{array}$ & $\begin{array}{l}\text { Departamento de Investigación y Conocimiento } \\
\text { para el Diseño }\end{array}$ \\
\hline https://www.azc.uam.mx/ & https://www.cyad.online/uam/ & http://investigacionyconocimiento.azc.uam.mx/ \\
\hline (c) $(\mathrm{B}) \Theta$ & \multicolumn{2}{|c|}{$\begin{array}{l}\text { Excepto si se señala otra cosa, la licencia del ítem se describe como } \\
\text { Atribución-NoComercial-Sin Derivadas } \\
\text { https://creativecommons.org/licenses/by-nc-nd/4.0/ }\end{array}$} \\
\hline
\end{tabular}

D.R. (C) 2012. Universidad Autónoma Metropolitana. Unidad Azcapotzalco (UAM Azcapotzalco). Se autoriza copiar y redistribuir el material en cualquier medio o formato, siempre y cuando se den los créditos de manera adecuada, no puede hacer uso del material con propósitos comerciales, si remezcla, transforma o crea a partir del material, no podrá distribuir el material modificado. Para cualquier otro uso, se requiere autorización expresa de la UAM Azcapotzalco. 
DOI: 10.24275/uama.7048.7676

CONCEPTOS DESARROLLADOS

- Ambiente

- Ciclo de vida

- Consumo

- Ecología

- Ecoeficiencia

- Ecosistema

- Impacto ambiental

- Resiliencia

- Sistema

- Sustentabilidad 
DOI: $10.24275 /$ uama.7048.7676

DESARROLLO DE CONCEPTOS POR:

MDI. Isaac Acosta Fuentes (coordinador)

Profesor-investigador del Departamento

Investigación y Conocimiento para el Diseño,

División CyAD, UAM-Azc.

MTRO. Marcio Dupont Caballero de Carranza

Profesor del Instituto Europeu de Diseño (IED)

en São Paulo, Brasil

MDI. Brenda García Parra

Profesora del Posgrado en Diseño Industrial,

UNAM

MDI. Sandra Luz Molina Mata

Profesora-investigadora del Departamento de

Investigación y Conocimiento para el Diseño,

UAM-Azc./ División CyAD 


\section{Sustentabilidad y diseño industrial}

El principal objetivo de este tema es describir el modo en que el diseño industrial se relaciona con los planteamientos de la sustentabilidad, que constituye un enfoque del desarrollo de la sociedad. Las teorias del desarrollo económico surgieron en la segunda mitad del siglo XX. Después de un largo proceso de estructuración y maduración, a finales de la decada de los años 80 estas teorías se sintetizan en la visión del desarrollo sustentable. La Organización de las Naciones Unidas (ONU), a través de su Comisión para el Medio Ambiente, es la instancia responsable de impulsar este enfoque, que no es el único. Se hablará del desarrollo sustentable o de sustentabilidad de manera indistinta.

La sustentabilidad propone que las sociedades actuales deben de satisfacer sus necesidades cabalmente, pero sin poner en entredicho las posibilidades para que las futuras generaciones tambien satisfagan sus propias necesidades. Esto significa que las generaciones actuales deben evitar el agotamiento de los recursos naturales y deben procurar mantener los procesos ambientales de forma tal que nuestro planeta continúe ofreciendo condiciones favorables a la reproducción de la vida.

Para lograr estos objetivos es necesario que exista equidad social y que las actividades económicas de toda la sociedad procuren el bienestar. En otras palabras, el desarrollo sustentable tiene tres pilares: la protección del ambiente, la equidad social y el desarrollo económico.

La sustentabilidad es un proceso y como tal demanda acciones permanentes. Ciertamente, implica una filosofía de la vida, pero no se trata solamente de un discurso. La sustentabilidad de un sistema social se mide y con ese objetivo se generan indicadores cuantitativos en los ámbitos ambiental, económico y social. En lo anterior, la premisa es que nuestro crecimiento 
económico no puede ser infinito, sino que tiene que acotarse a límites naturales y sociales.

Por otro lado, el diseño industrial es una disciplina resultado de la modernidad, cuyo desarrollo ha traído diversos beneficios sociales: muchos productos de diseño han mejorado la calidad de vida de los usuarios y comunidades que los emplean, mientras que la disciplina se ha constituido en un valioso instrumento de competitividad económica para las empresas manufactureras. A través del diseño se ha dado impulso a la innovación en términos de la interacción de las personas con los objetos, y de este modo, la cultura material se ha enriquecido.

Ahora bien, este impulso a la sociedad de consumo, que disciplinas como el diseño industrial ha fomentado, no es del todo favorable para el desarrollo de las sociedades. Hoy en día, el impacto ambiental de la producción de mercancías tiene alcances globales, debido a que los procesos de extracción de materiales para la producción de materias primas y la transformación de esas materias primas en productos ocurren en diferentes partes del mundo.

Los efectos sociales y ambientales de estos procesos de extracción y transformación no han sido contemplados como un aspecto de relevancia para el proceso de diseño. No obstante, durante ese proceso proyectual de los productos ocurren buena parte de las decisiones que originan impactos negativos en los medios ecológicos y las sociedades que los habitan.

Además, las etapas de distribución, consumo y desecho de los productos implican consumos energéticos, empleo y desgaste de recursos y espacios $y$, finalmente, el confinamiento de los residuos. Una parte importante de los efectos negativos de esas etapas, a lo largo de la vida de los productos, podrían limitarse o eliminarse si se prevén soluciones desde el proceso de diseño de los mismos.

En consecuencia, los diseñadores industriales deben reconocer y satisfacer un conjunto de requerimientos que den viabilidad a los productos en cuanto a criterios ambientales y socioeconómicos. Estos requerimientos tienen una relación con fenómenos que pueden ser cuantificados, como es la cantidad de energía consumida de modo eficiente; el total y tipo de emisiones generadas por contaminantes; o bien, de orden cualitativo pero verificable, como la existencia y accesibilidad de sistemas de recuperación de materiales para su reciclaje. 
Ninguno de estos parámetros es suficiente por sí mismo, sino que se requiere un enfoque integral de los tres ámbitos (ambiente, sociedad y economía) que se traduzca en indicadores controlables para apoyar el proceso de diseño de productos.

El diseño se caracteriza por ser una actividad innovadora que propone soluciones, sobre todo en cuanto al modo en que se resuelven necesidades de las personas y la implementación del desarrollo tecnológico en la vida cotidiana. En este terreno, el diseño ofrece una aportación al desarrollo sustentable, pues se trata de una disciplina que se cuenta entre las responsables de la proyección del ambiente artificial.

En resumen, el desarrollo sustentable plantea al diseño industrial el reto de desarrollar procesos proyectuales que se apoyen en requerimientos ambientales, económicos y sociales, y que se generen instrumentos de evaluación cuantitativa y cualitativa del desempeño de los productos, en todas las etapas de su vida.

A su vez, el diseño debe enfocarse con una visión integral del ciclo de vida del producto y debe orientarse hacia la limitacion del impacto ambiental, la recuperación del medio ecológico y la calidad de vida de los usuarios. Es decir, la innovación generada en el diseño de productos debe promover estilos de vida viables en el corto y largo plazo.

En principio, señalamos que el desarrollo sustentable implica diferentes visiones. Una forma de esquematizar estas diferencias es presentar dos grandes enfoques del tema: la sustentabilidad fuerte y la sustentabilidad débil. En términos del primer enfoque, el desarrollo socioeconómico tiene un derrotero en el ámbito ecológico pues cuando se agotan los recursos naturales no renovables no hay modo de recuperar sus servicios y, por lo tanto, no existe un mecanismo viable de sustitución de valores naturales por valores económicos.

En el segundo enfoque, la sustentabilidad débil, los instrumentos del sistema económico de mercado como los precios de los recursos y las sanciones por daños ambientales, constituyen mecanismos propicios para orientar las actividades productivas y los estilos de vida hacia un sistema equilibrado. En esta visión los valores naturales son sustituibles por valores económicos.

Estas dos posturas, con sus matices, mantienen un debate hasta ahora vigente. En el campo del diseño estas posturas no se han traducido en una fuente 
de profunda discusión, pero sí existen diferentes concepciones relativas al grado de alcance del diseño en cuanto a los objetivos de la sustentabilidad.

Uno de los ejes de las diferentes visiones dentro del diseño hacia la sustentabilidad es la discusión de la viabilidad financiera versus la viabilidad social y ambiental del desarrollo de productos. Atendiendo a este eventual conflicto, algunos diseñadores creen que là disciplina debe enfocarse a limitar el impacto ambiental de los productos, mientras que otros proponen un cambio de los objetivos del proceso de diseño orientándose hacia la configuración de nuevos entornos sociales y estilos de vida sustentables.

No obstante, salvo el medio académico, estas discusiones suelen ser obviadas debido a una actitud pragmática de una parte de la comunidad de diseño, con perjuicio para la calidad de los productos de diseño desarrollados al amparo de concepciones de escasa profundidad. De ahí la importancia de abrir debates, socializar experiencias y construir redes de diseño pensando en los problemas de la sustentabilidad.

Lo anterior es una condición indispensable para la relación del desarrollo sustentable y el diseño: el fomento del trabajo interdisciplinario como base de un diálogo disciplinar y el aliento a la integración de procesos de desarrollo de productos con perfil multidisciplinario.

Isaac Acosta Fuentes 


\section{Ambiente}

\section{DEFINICIÓN DicCIONARIO RAE (DENOTACIÓN)}

(Del lat. ambǐens, -entis, que rodea o cerca). 1. adj. Dicho de un fluido: Que rodea un cuerpo. 2. m. Aire o atmósfera. 3. m. Condiciones o circunstancias físicas, sociales, económicas, etc., de un lugar, de una reunión, de una colectividad o de una época. 4. m. Grupo, estrato o sector social. Ambientes intelectuales, populares, aristocráticos. 5. m. Actitud de un grupo social o de un conjunto de personas respecto de alguien o algo. Juan tiene buen ambiente entre sus colegas. La propuesta encontró mal ambiente. 6. m. Pint. Efecto de la perspectiva aérea que presta corporeidad a lo pintado y finge las distancias. 7. m. Am. Habitación de una casa.

\section{DEsARROLLO O CONSTRUCCIÓN DEL CONCEPTO}

Los elementos naturales y artificiales que reunidos en el marco de un territorio se interrelacionan para constituir el medio que posibilita la existencia y el desarrollo de los organismos.

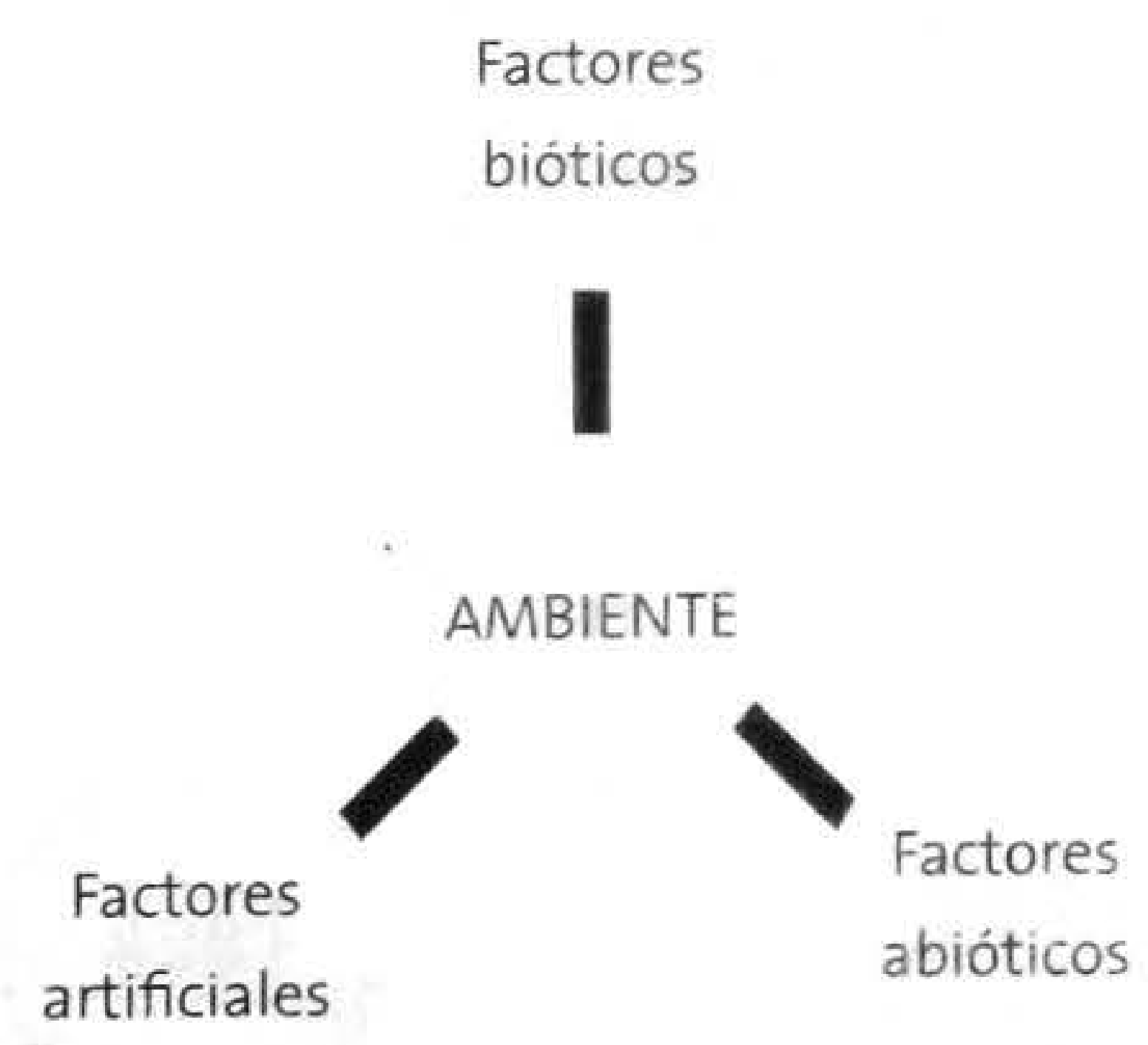

Figura 6.1. El ambiente y sus diferentes factores. Elaboración propia.
El conocimiento del entorno es fundamental para el diseñador, pues sus acciones están encaminadas a configurar los elementos artificiales, los cuales mantienen una constante interacción con los factores bióticos y abióticos del ambiente.

\section{Conceptos}

"Conjunto de elementos naturales, artificiales o creados por el hombre, físicos, quimicos y biológicos que posibilitan la existencia, transformación y desarrollo de organismos vivos" (Sabsay, citado por Godoy, 2005:21).

\section{USOS O ACEPCIONES (ACLARACIONES)}

\section{Ambiente natural}

"Es un conjunto de agentes físicos, químicos, biológicos y sociales susceptibles de provocar un efecto directo o indirecto sobre los aspectos humanos y, en general, sobre los seres vivientes. Puede asociarse con el entorno, compuesto por elementos interdependientes, interrelacionados y cohesionados" (Godoy, 2005:22). Ambiente artificial (ambiente humano) "Entorno natural que ha sido alterado artificialmente por el hombre y su cultura. Está constituido por tres factores básicos:

- Lo abiótico (tierra, atmósfera, aire, sonido, clima, olores, sabores).

- Lo biótico (animales domésticos, plantas, bacterias, virus).

- Los factores antropogénicos (higiene, estética, cultura, religión, deporte, política, etc.). Este ambiente es considerado como un ecosistema subordinado de la biosfera, 
que afecta la estabilidad de los sistemas naturales vecinos" (Galván, 2007:24).

\section{SINÓNIMOS}

Entorno, medio, atmósfera, ámbito, esfera, hábitat, biósfera, ecoesfera.

\section{ANTÓNIMO}

No existe.

\section{EJEMPLOS}

Ambiente artificial construido por el hombre, la llamada tecnoesfera, consumiendo el ambiente natural o ecoesfera, que no tiene la misma capacidad o velocidad de reproducción.

\section{Bibliografía}

GALVÁN Meraz, Francisco Javier (2007), Diccionario ambiental y asignaturas afines, México, Ediciones Mundi Prensa.

Godoy, Emiliano V. (2005), Diccionario de Ecología, Argentina: Valletta Ediciones.

MANzINI, Ezio (1992), Artefactos: hacia una ecología del ambiente artificial, Madrid: Celeste ediciones.

\section{Ciclo de vida}

\section{Definición Diccionario RAE (DENOTACIÓN)}

Ciclo (Del lat. cyclus, y este del gr. $x v \dot{x} \lambda o s$, círculo). 1. $m$. Periodo de tiempo o cierto número de años que, acabados, se vuelven a contar de nuevo. 2. m. Serie de fases por las que pasa un fenómeno periódico. 3. m. Conjunto de una serie de fenómenos $\mathrm{u}$ operaciones que se repiten ordenadamente. Ciclo de un motor de explosión, de una máquina herramienta, de la corriente eléctrica. 4. m. Serie de conferencias u otros actos de carácter cultural relacionados entre sí, generalmente por el tema. 5. m. Cada uno de los bloques de cursos en que se divide un plan de estudios. 6. m. Conjunto de tradiciones épicas concernientes a determinado periodo de tiempo, a un grupo de sucesos o a un personaje heroico. El ciclo troyano, el ciclo bretón, el ciclo del rey Artús o Arturo. 7. m. Bot. Cada una de las espiras que se forman alrededor del tallo, los puntos de inserción de las hojas. 8. m. Quím. Molécula cerrada con un número definido de átomos, como el benceno. 9. m. Cuba. bicicleta.

\section{DESARROLLO O CONSTRUCCIÓN DEL CONCEPTO}

El conjunto de etapas por las que atraviesa la existencia de un producto, desde la obtención de las materias primas para su fabricación, hasta su retiro o reciclaje (total o parcial). En el siguiente

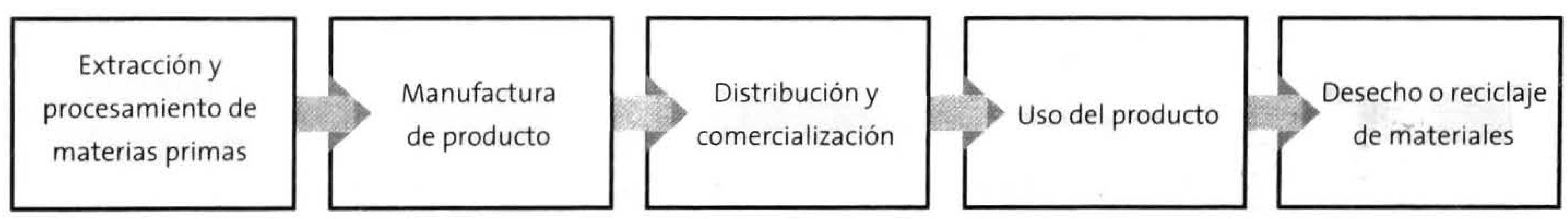

Figura 6.2. Etapas del ciclo de vida de un producto. Elaboración propia. 
diagrama se pueden observar las principales etapas del ciclo de vida de un producto.

El diseño de un producto implica la determinación de requerimientos específicos para cada etapa de la vida del producto con la finalidad de fomentar el carácter cíclico de la utilización de los materiales empleados.

\section{Conceptos}

Análisis de ciclo de vida (ACU)

"Herramienta de gestión ambiental que estudia los aspectos ambientales y los impactos potenciales a lo largo de la vida de un producto, proceso o actividad, desde la adquisición de las materias primas hasta la producción, uso y eliminación".

"Las fases de elaboración de ACV a grandes rasgos son: recopilación de las entradas y salidas relevantes de un sistema (energía, materias utilizadas y residuos vertidos al medio), evolución de los potenciales impactos ambientales asociados con estas entradas y salidas (uso de recursos, efectos sobre la salud humana, consecuencias ecológicas, etc.) y, finalmente, desarrollo de prácticas de mejora ambiental" (Galván, 2007:25).

\section{USOS O ACEPCIONES (ACLARACIONES)}

La acepción del ciclo de vida, que antes se limitaba al mundo natural, ahora se extiende al mundo industrial, con la ecología industrial, análisis del ciclo de vida de productos, con la responsabilidad del productor. Esa responsabilidad se incrementa en los países emergentes, donde empiezan a surgir nuevas politicas-leyes de residuos sólidos. Estas nuevas reglas exigen que todos los participantes: gobierno, productores, consumidores se hagan responsables del ciclo de vida del producto en sus diferentes fases, desde la extracción hasta el desecho.

Dependiendo del enfoque, el proceso de diseño puede estar dirigido a propiciar un ciclo "abierto" (de la cuna a la tumba - Cradle to Grave), o "cerrado" (de la cuna a la cuna - Cradle to Cradle). El ciclo abierto no considera la re-integración de los elementos de un producto o solución de diseño nuevamente a un sistema biológico o industrial, que sí es propiciado por un ciclo cerrado.

En inglés se le conoce como "Life Cycle", por lo que la abreviatura para el análisis del ciclo de vida es conocido como Life Cycle Assesment (ICA), ampliamente identificado.

\section{SINÓNIMO}

Visión holística del producto.

\section{ANTÓNIMOS}

Ciclo parcial, ciclo incompleto, ciclo individual, ciclo aislado.

\section{EJEMPLOS}

- Ciclo de vida de los productos electrónicos, causantes de grandes contaminaciones ambientales debido a metales tóxicos y materiales que no pueden ser reciclados o reusados.

- Ciclo de productos comparativos (bolsas de tela vs. bolsas de plástico).

\section{Bibliografía}

EIGner, M. y Stelzer (2009), Product Lifecycle Management: Ein Leitfaden fur Product 
Development und Life Cycle Management, Springer. German edition.

FIKSEL, J. (2009), Design for Environment: A Guide to Sustainable Product Development, 2nd edition. McGraw-Hill Professional, EU.

Galván Meras, Francisco Javier (2007), Diccionario ambiental y asignaturas afines, México: Mundi prensa.

McDonough, William y Braungart (2002), Cradle to cradle: Remaking the way we make things, New York: North Point Press.

Ny, Henrik, J.P. (2006), "Sustainability constraints as system boundaries: An approach to making life cycle analysis strategic", Journal of Industrial Ecology, Vol. 10, Núm. 1-2, pp. 61-77.

UNEP. DRAFT UNEP (2008), Life Cycle Management Training Kit LCM as part of Good Practice. Life Cycle Management Training - Outline. I. http://www.oecd.org/document/19/0,3746,en_ 2649_34281_35158227_1_1_1_1,00. (Consultado 23 nov. 2011).

\section{Consumo}

\section{DEFINICIÓN DicCiONARIO RAE (DENOTACIÓN)}

Consumo. 1. m. Acción y efecto de consumir (comestibles y otros géneros de vida efímera). 2. m. Acción y efecto de consumir (gastar energía). 3. m. ant. Extinción de caudales, de juros, libranzas o créditos contra la Real Hacienda. 4. m. pl. Impuesto municipal sobre los comestibles y otros géneros que se introducen en una población para venderlos o consumirlos en ella.
Consumir (Del lat. consumerre). 1. tr. Destruir, extinguir. U. t. c. prnl. 2. tr. Utilizar comestibles $\mathrm{u}$ otros bienes para satisfacer necesidades o deseos. 3. tr. Gastar energía o un producto energético. 4. tr. Dicho de un sacerdote: Recibir o tomar la comunión en la misa. U.t. c. intr. 5. tr. coloq. Desazonar, apurar, afligir. U. t. c. prnl. 6. tr. ant. Dicho de los legítimamente casados: consumar. 7. prnl. Col. y C. Rica. Zambullirse en el agua.

\section{DESARROLLO O CONSTRUCCIÓN DEL CONCEPTO}

El consumo es el acto con el que culmina el proceso económico y que posibilita el sustento y reproducción de los individuos y las sociedades. Como acto económico corresponde con la satisfacción de necesidades, individuales y colectivas, que poseen una dimensión histórica (ver Figura 6.3).

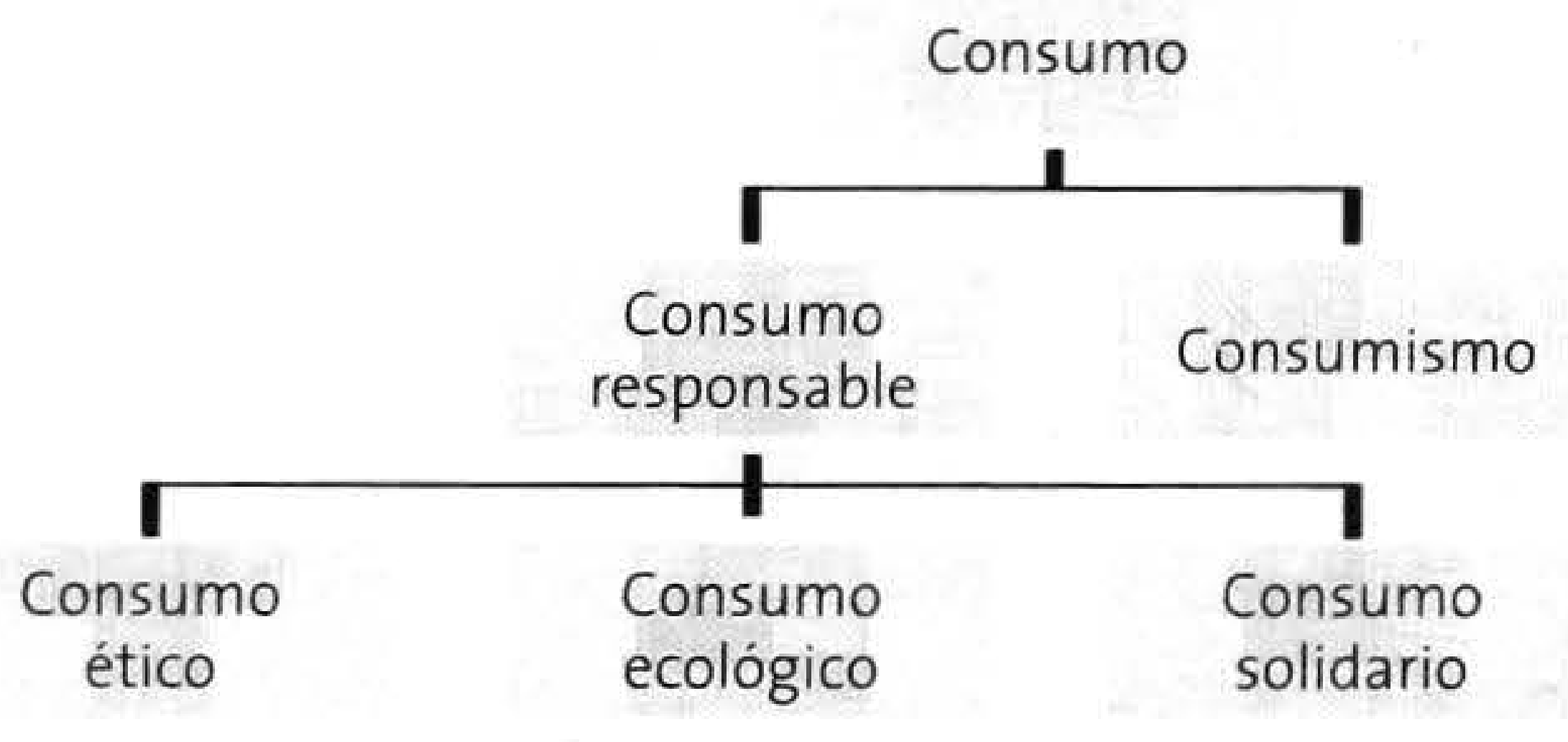

Figura 6.3. Dimensiones del consumo. Elaboración propia.

El diseñador tiene un papel muy importante pues los objetos fomentan hábitos de consumo que pueden ser compatibles con la sustentabilidad o, por el contrario, estilos de vida negativos, como el consumismo.

\section{Conceptos}

"Acto final del proceso económico, que consiste en la utilización personal y directa de los bienes 
y servicios productivos para satisfacer necesidades humanas. El consumo puede ser tangible (consumo de alimentos) o intangible (consumo de servicios); puede ser inmediato el consumo, en el corto o en el largo plazo" (Zorrilla, 1994:41)

"Proceso de obtener utilidad de una mercancía o servicio. En sentido más general, describe el proceso de adquisición de mercancías y servicios para obtener satisfacciones directas de ellas; o indica la cantidad de gasto que se realiza en ellas... En economía 'consumo' no implica necesariamente la destrucción física de la mercancía 'consumida'. Los alimentos se consumen, pero también se consumen cuadros, que no quedan destruidos al rendir una utilidad o satisfacción. El consumo no tiene por que ser necesariamente un proceso tangible; puede decirse que una audiencia 'consume' los servicios de los actores o músicos" (Seldon, 1980:143).

\section{USOS O ACEPCIONES (ACLARACIONES)}

"El concepto de consumo responsable es muy amplio, como lo es la propia actividad de consumir. Podemos, sin embargo, sintetizarlo en tres bloques:

1. Un consumo ético, en el que se introduzcan valores como una variante importante a la hora de consumir o de optar por un producto. Se hace especial énfasis en la austeridad como un valor con relación a la reducción para un consumo ecológico, pero también frente al crecimiento económico desenfrenado y al consumismo como forma de alcanzar el bienestar y la felicidad.

2. Un consumo ecológico, que incluye, por este orden, las famosas 'erres' del movimiento ecologista: Reducir, Reutilizar y Reciclar, pero en el que también se incluyen elementos tan imprescindibles como la agricultura y ganadería ecológicas, la opción por la producción artesanal, etc.

3. Un consumo social o solidario, en el que entraria también el comercio justo, es decir, el consumo en lo que se refiere a las relaciones sociales y condiciones laborales en las que se ha elaborado un producto o producido un servicio. Se trata de pagar lo justo por el trabajo realizado, tanto a personas de otros países como a las más cercanas en el ámbito local; se trata de eliminar la discriminación, ya sea a causa del color de la piel o por diferente origen, o por razón de género o religión; se trata de potenciar alternativas sociales y de integración y de procurar un nuevo orden económico internacional" (www.consumoresponsable.com/ portada. Consultado 8 de junio de 2011).

\section{SINÓNIMOS}

Compra, adquisición, utilización, desgaste.

\section{ANTÓNIMO}

Venta.

\section{EJEMPLOS}

Consumo de productos y servicios.

\section{BibLiografía}

JHA, Raghbendra (2006), Environmental sustainability: a consumption approach, Londres: Routledge. 
Mansvelt, Juliana (2005), Geographies of consumption, Londres: SAGE, 2005.

PORRIT, Jonathan (2007), Capitalism: As If the World Matters (Revised Edition, EUA), Earthscan Publications.

SELDON, Arthur; F.G. Pennance (1980),

Diccionario de economia, España: Oikos-

tau S. A. ediciones, $3^{\text {a }}$. Edición castellana.

ZORRILLA Arena, Santiago y José Silvestre Méndez

(1994), Diccionario de economía, México:

Limusa Noriega Editores, 2 edición.

ZUKIN, Sharon (2005), Point of purchase:

how shopping changed American

culture. New York: Routledge.

http://www.consumoresponsable.com/portada. htm (consultado 8 de junio de 2011, 12:30 p.m.) http://www.oecd.org/topic/o,3699,en 2649_34289_1_1_1_1337465,00.html

\section{Ecologia}

\section{Definición DicCionario RAE (DENOtACIÓN)}

(De eco-y -logía). 1. f. Ciencia que estudia las relaciones de los seres vivos entre sí y con su entorno. 2. f. Parte de la sociología que estudia la relación entre los grupos humanos y su ambiente, tanto físico como social. 3. f. Defensa y protección de la naturaleza y del medio ambiente.

\section{DESARROLLO O CONSTRUCCIÓN DEL CONCEPTO}

La ecología es la disciplina que estudia el sistema de interrelaciones construidas entre los organismos vivos y los factores ambientales que posibilitan su existencia y reproducción (ver Figura 6.4).

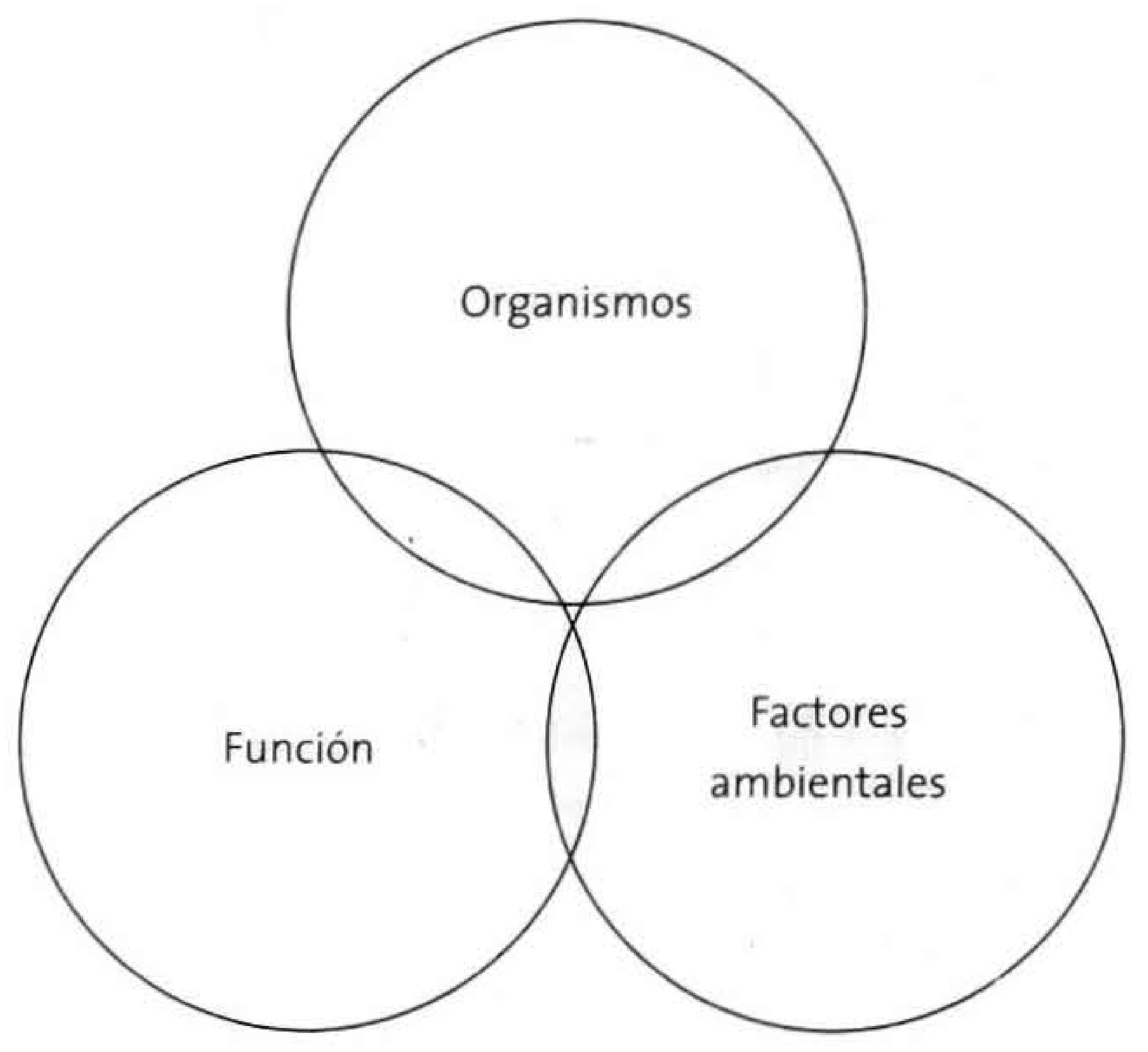

Figura 6.4. La Ecología y sus diferentes componentes. Elaboración propia.

Mediante el proceso de diseño se establecen las relaciones entre el medio ecológico y el mundo artificial. Al diseñarse un producto se debe considerar la forma en que éste afectará al entorno, tomando en cuenta los impactos en los organismos vivos, los factores ambientales y sus mutuas relaciones naturales.

\section{Conceptos}

"Estudio de las relaciones entre los organismos vivos y su medio ambiente" (Allaby, 1984:141).

"Ciencia de la relación de los organismos con su medio ambiente, tanto el animado como el inanimado; es la ciencia de la 'economía doméstica' de las plantas y animales" (Hesse citado por Godoy, 2005:75).

"Es el estudio de la 'morfología de la vida colectiva', en sus aspectos tanto estáticos como dinámicos. El objeto de la investigación es la comunidad, cuya forma y desarrollo se estudian 
con especial referencia a los factores del medio ambiente que sirven de límite o apoyo".

"Se ocupa de cómo los seres que crecen y se multiplican, se mantienen en un medio ambiente constantemente en cambio, pero siempre limitado. Ciencia de las correlaciones entre los organismos que conviven en una y la misma localidad y sus adaptaciones al medio ambiente" (Haeckel, citado por Godoy, 2005:75).

"Disciplina que trata del estudio de las interacciones de los seres vivos entre si y con su medio ambiente; su objetivo central es el de describir los principios que gobiernan esas interrelaciones".

"El medio, la función y el organismo constituyen juntos lo que puede ser llamado la tríada biológica fundamental. Esa triada tiene que ser estudiada como un todo completo, y este estudio es lo que se quiere decir con este término" (Bews, citado Godoy, 2005:75).

"Proviene del grupo y se compone de dos vocablos cuyo significado es el tratado o estudio de la casa o lugar donde se habita. Se ocupa del estudio de todos los elementos que componen el planeta Tierra y de la relación entre ellos. En general, se refiere al género de lo humano, de las plantas, animales y microorganismos que conviven en forma independiente. Incluye el fenómeno de la energía y los ciclos de la materia en las aguas y en el aire" (Piña, citado por Godoy, 2005:75).

"Ciencia capaz de resolver las tres crisis que se consideran fundamentales: la crisis de recursos, la del aumento de la población y la contaminación. Su punto de partida es considerar que la contaminación obedece a que la industria ha sido insuficiente para eliminar los desechos de su propia actividad. En sintesis, es la ciencia que se ocupa de las relaciones de interdependencia entre los seres vivientes y el espacio vital. Estudia la estructura y el funcionamiento de la naturaleza en cuanto sistema organizado, en su totalidad" (Godoy, 2005:75).

\section{USOS O ACEPCIONES (ACLARACIONES)}

Ecología de poblaciones

"Estudio de los factores que afectan al número de individuos de una población cualquiera que se encuentra en un área específica durante un periodo de tiempo".

Ecologia de producción

"Estudio de los biomas en términos de la producción y distribución de alimentos; $\mathrm{y}$, por tanto, del flujo de energía dentro del mismo".

Ecologia industrial

"Es un sistema donde se optimiza el consumo de energía y de materiales, se minimiza la generación de descargas y se favorece la reutilización de residuos de un proceso como materia prima para otros procesos. Es el diseño de sistemas industriales ecoeficientes, con la participación de una o más empresas, que utilizan o imitan los patrones cíclicos de los flujos de materiales y energía que existen en los ecosistemas naturales" (Godoy, 2005:75).

Un producto "ecológico" no es aquel que no tiene impacto alguno en el medio. Actualmente se denomina así a aquel que reduce su impacto ambiental, aunque etimológicamente sea incorrecto, pues la raíz de la palabra indica únicamente su relación con el estudio del ambiente. 


\section{SINÓNIMOS}

Ecológico, ecologista, ecosistema.

\section{ANTÓNIMOS}

Contaminante, tecnocéntrico, industrial.

\section{EJEMPLOS}

Ecología del organismo, ecología de las populaciones, ecología de las comunidades.

\section{Bibliografía}

AlLABY, Michael (1984). Diccionario del medio ambiente, España: Ediciones Pirámide.

GaLVÁN Meraz, Francisco Javier (2007), Diccionario ambiental y asignaturas afines, México, Mundi Prensa.

Godoy, Emiliano V. (2005), Diccionario de Ecología, Argentina: Valletta Ediciones.

SEMARNAT (2007), ¿Y el medio ambiente? Problemas en México y en el mundo. México.

Session, George (1995), Deep Ecology for the 21st century, Boston: Ed. Shambhala. http://www.oecd.org/topic/0,3699,en_2649_ 34281_1_1_1_1_37465,00.html Consultado 24 de agosto de 2011.

\section{Ecoeficiencia}

\section{DESARROLLO O CONSTRUCCIÓN DEL CONCEPTO}

La ecoeficiencia es una característica de los sistemas productivos que mantienen una relación equilibrada con los ecosistemas que los sustentan, ya que no alteran la capacidad productiva primaria de los mismos. En otras palabras, un sistema productivo ecoeficiente tiene una tasa de explotación ecológica que no agota las capacidades de carga y resiliencia del medio ecológico, minimiza la explotación y agotamiento de recursos, así como reduce la cantidad de emisiones y desechos al ambiente.

La ecoeficiencia es una situación meta para el diseño ecológico. El proceso de diseño de productos debe considerar indicadores que le permitan al diseñador tomar las mejores decisiones en cuanto a la configuración de los productos, de forma tal que la relación entre los recursos extraídos del medio natural y los impactos generados por el consumo energético y las emisiones por desechos no excedan las capacidades regenerativas del propio medio ecológico (ver Figura 6.5).

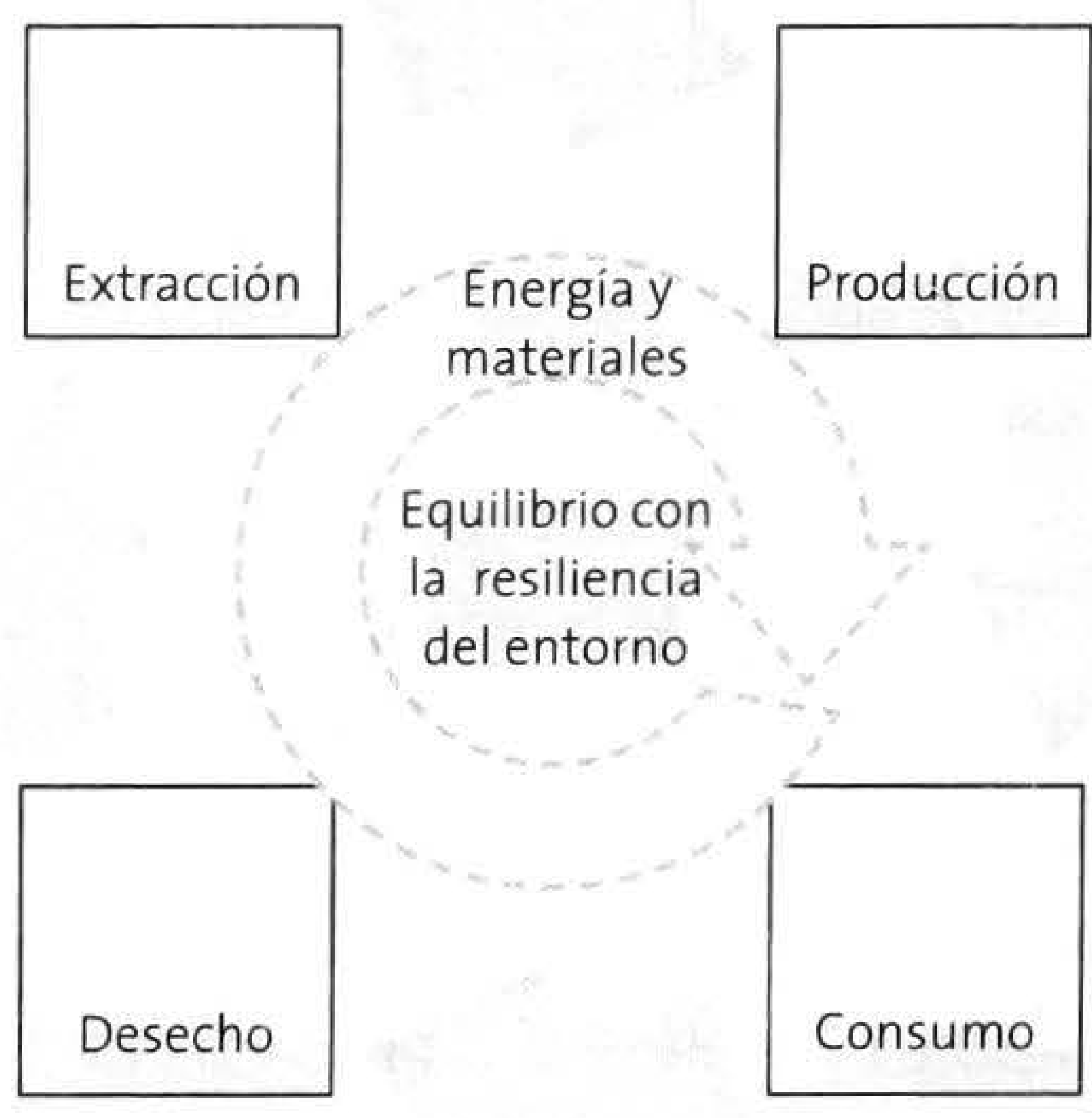

Figura 6.5. Criterios de la ecoeficiencia. Elaboración propia.

\section{USOS Ó ACEPCIONES (ACLARACIONES)}

Eficiencia ecológica

"La dimensión (ratio) entre flujos de energía 
medidos en diferentes puntos en una cadena alimenticia, usualmente expresados como porcentaje. Muchas aproximaciones han sido distinguidas por diferenciar aspectos diversos (por ejemplo "intake", asimilación y producción). Las dos principales categorias estudiadas de eficiencia son: a) la transferencia de energia entre diferentes niveles tróficos y b) la transferencia de energía en un mismo nivel trófico" (traducción libre, Allaby, 2005:145).

"De acuerdo con la Ecologia Industrial, es el conjunto de objetivos orientados al mejor aprovechamiento de los recursos (mediante un menor uso de estos pero con mayor eficiencia en su fabricación o utilización) y a la reducción de la contaminación a lo largo del ciclo de vida de los productos sin descuidar cualidades técnicas funcionales y económicas" (García, 2008:71).

\section{SINÓNIMO}

Eficiencia ecológica

\section{ANTÓNIMO}

Ineficiencia ecológica

\section{EJEMPLOS}

- La explotación forestal con criterios ecológicos persigue lograr una ecoeficiencia en términos de garantizar la disposición permanente de los recursos madereros a lo largo del tiempo.

- El reciclaje busca, entre otros objetivos, una ecoeficiencia en cuanto al uso productivo de los materiales empleados en la manufactura.
- El ahorro energético generado con la sustitución de focos incandescentes por otros sistemas de menor consumo es una medida que puede ser caracterizada como ecoeficiente.

\section{Bibliografía}

Díaz Coutiño, R.; Desarrollo sustentable una oportunidad para la vida, México, Mc Graw Hill, 2011

Dictionary of Ecology, ed. By Michael Allaby; Oxford University Press, Third Edition, 2005.

García Parra, Brenda; Ecodiseño. Nueva herramienta para la sustentabilidad, Editorial Designio, México, 2008.

\section{Ecosistema}

\section{DEFINICIÓN DicCIONARIO RAE (DENOTACIÓN)}

Comunidad de los seres vivos cuyos procesos vitales se relacionan entre sí y se desarrollan en función de los factores físicos de un mismo ambiente.

\section{DESARROLLO O CONSTRUCCIÓN DEL CONCEPTO}

El concepto de ecosistema implica la serie de relaciones entre los elementos bioticos y abioticos en un ambiente determinado. Estas relaciones pueden verse alteradas, dando origen a desequilibrios.

Los elementos que estructuran un ecosistema, bióticos y abióticos, guardan relaciones dinámicas pero equilibradas. Estas relaciones se expresan como flujos metabólicos de materia y energía. 


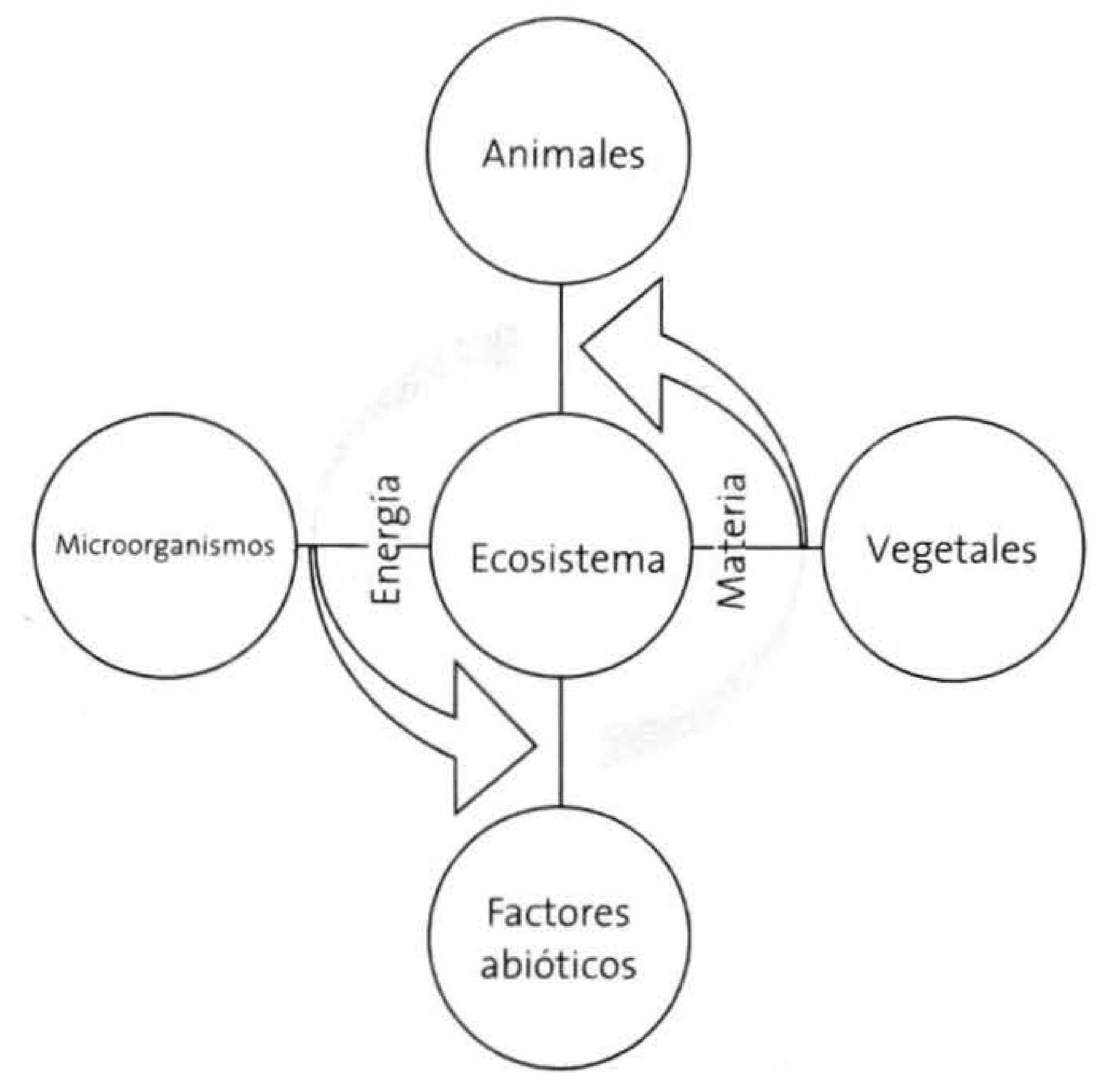

Figura 6.6. Elementos que conforman ecosistemas. Elaboración propia.

Las acciones de los seres humanos pueden alterar la situación de equilibrio de los ecosistemas. En particular, la producción industrial ha sido un importante mecanismo de afectación a los ecosistemas.

"Complejo dinámico de comunidades vegetales, animales y de microorganismos y su medio no viviente que interactúan como una unidad funcional. Máxima unidad funcional de la naturaleza en la Tierra; es un sistema que tiene circulación de materia y energía; es funcional y esta funcionalidad está dada sobre la base de su biodiversidad; es rico en información y guarda en él las estructuras de cadenas alimenticias básicas para el desarrollo de la vida en la Tierra; el ecosistema es una delicada unidad de funciones biológicas sorprendentes que guardan un intrínseco equilibrio entre los factores bióticos y abióticos presentes en él." (Godoy, 2005:78).
"Sistema abierto integrado por todos los organismos vivos (incluyendo al hombre) y los elementos no vivientes de un sector ambiental definido en el tiempo y en el espacio, cuyas propiedades globales de funcionamiento y auto regulación derivan de las interacciones entre sus componentes, tanto pertenecientes a los sistemas naturales como aquellos modificados u organizados por el hombre mismo". (Sánchez, 1982:43).

\section{USOS O ACEPCIONES (ACLARACIONES)}

Desde la perspectiva de la ecología el ecosistema es una unidad de estudio, que permite analizar una serie de relaciones de los seres en su medio. Esto permite cuantificar información sobre los impactos a los que está sujeto.

\section{SINÓNIMO}

Sistema ecológico

Algunas veces se equipara con el concepto de hábitat o medio ambiente, sin embargo esto no es preciso.

\section{ANTÓNIMO}

No existe

\section{EJEMPLOS}

La ciudad como un ecosistema urbano hace referencia a la idea de lograr un desarrollo urbano coherentemente integrado con la existencia de un medio ecológico sano.

\section{Bibliografía}

DíAz Coutiño, R.; Desarrollo Sustentable una 
impacto ambiental de

las acciones antrópicas

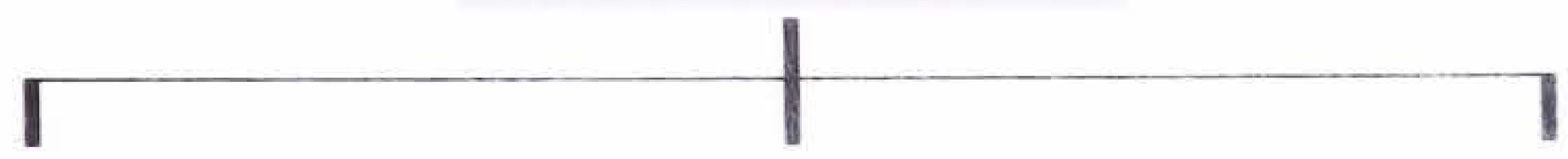

Efectos medibles sobre los factores ambientales y los recursos disponibles en el corto y largo plazo
Efectos cualitativos sobre las interrelaciones de los factores ambientales y bióticos
Pérdida de capacidad de resiliencia del medio natural

Figura 6.7. Impacto ambiental de las acciones antrópicas. Elaboración propia.

Oportunidad para la Vida, Macgraw Hill, México, 2011.

Godoy, Emiliano V.; Diccionario de Ecologia, Valletta Ediciones, Argentina, 2005.

Sánchez, Vicente; Guiza, Beatriz; Glosario de términos sobre medio ambiente, 1ed., El Colegio de México, México, 1982.

\section{Impacto ambiental}

\section{Definición Diccionario RAE (DENOTACIÓN)}

Impacto (Del lat. tardío impactus). 1. m. Choque de un proyectil o de otro objeto contra algo. 2. m. Huella o señal que deja. 3. m. Efecto de una fuerza aplicada bruscamente. 4. m. Golpe emocional producido por una noticia desconcertante. 5. m. Efecto producido en la opinión pública por un acontecimiento, una disposición de la autoridad, una noticia, una catástrofe, etc.

Ambiental. 1. m. Conjunto de posibles efectos negativos sobre el medio ambiente de una modificación del entorno natural, como consecuencia de obras u otras actividades.

\section{DESARROLLO O CONSTRUCCIÓN DEL CONCEPTO}

El conjunto de efectos ocasionados en el ambiente natural como consecuencia, principalmente, de las acciones humanas con relación a la construcción de obras, la producción, el desecho, las emisiones tóxicas, etc. El impacto ambiental es cuantitativo (medible) y cualitativo, en cuanto a la calidad de los factores ambientales y de los recursos.

La estimación del impacto ambiental de un producto es una tarea a contemplarse desde el proceso de su diseño. Para llevar a cabo esta tarea un instrumento como el Análisis de Ciclo de Vida tiene gran utilidad

\section{Conceptos}

"Modificación del ambiente ocasionado por la acción del hombre a la naturaleza. Se dice que hay impacto ambiental cuando una acción o actividad produce una alteración favorable o desfavorable en el medio o en alguno de los componentes del medio" (Galván, 2007:151).

\section{USOS O ACEPCIONES (ACLARACIONES) Impacto ambiental acumulativo}


"Efecto en el ambiente que resulta del incremento de los impactos de acciones particulares ocasionado por la interacción con otros que se efectuaron en el pasado o que están ocurriendo en el presente".

Impacto ambiental irreversible

"Es aquel que por la naturaleza de la alteración, no permitirá que las condiciones originales se restablezcan".

Impacto ambiental residual

"Impacto que persiste después de la aplicación de medidas de mitigación".

Impacto significativo o relevante

"Aquel que resulta de la acción del hombre o de la naturaleza que provoca alteraciones en los ecosistemas y sus recursos naturales o en la salud, obstaculizando la existencia y desarrollo del hombre y de los demás seres vivos, así como la continuidad de los procesos naturales".

\section{Impacto ambiental sinérgico}

"Aquel que se produce cuando el efecto conjunto de la presencia simultánea de varias acciones supone una incidencia ambiental mayor que la suma de las incidencias individuales contempladas aisladamente".

Impacto ambiental negativo

"Es el impacto ambiental cuyo efecto se traduce en pérdida de valor naturalístico, estéticocultural, paisajístico, de productividad ecológica o en aumento de los perjuicios derivados de la contaminación, de la erosión o colmatación y demás riesgos ambientales en discordancia con la estructura ecológico-geográfica, el carácter y la personalidad de una zona determinada" (Galván, 2007:151-152).

\section{SINÓNIMOS}

Choque, colisión, golpe, afectación, efecto negativo, resultante de salidas negativas de un sistema o evento, deterioro.

\section{ANTÓNIMOS}

Resistencia, rigidez, beneficio.

\section{EJEMPLOS}

- Impacto ambiental causado por el desastre petrolero en el Golfo de México.

- Problemas ambientales específicos relacionados a fases puntuales del ciclo de vida de un producto.

- Producción de un empaque: impactos en la impresión, recorte de papel, etc. Traducidos en los problemas ambientales causados (lluvia ácida, etcétera).

\section{Bibliografía}

GalvÁn Meraz, Francisco Javier (2007), Diccionario ambiental y asignaturas afines, Ediciones Mundi Prensa, México.

SEMARNAT (2007), ¿Y el medio ambiente? Problemas en México y en el mundo. México.

\section{Resiliencia}

\section{Definición Diccionario RAE (DENOtACIÓN)}

Resiliencia. 1. f. Psicol. Capacidad humana de asumir con flexibilidad situaciones límite y sobreponerse a ellas. 2. f. Mec. Capacidad de un material elástico para absorber y almacenar energía de deformación. 


\section{DESARROLLO O CONSTRUCCIÓN DEL CONCEPTO}

Capacidad de un sistema natural para recuperarse de los impactos negativos causados por agentes externos y reconstituir sus interrelaciones estables.

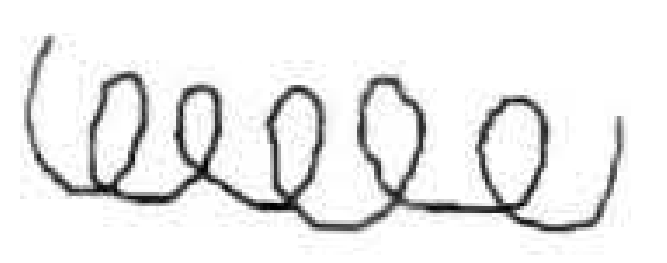

Estado Inicial

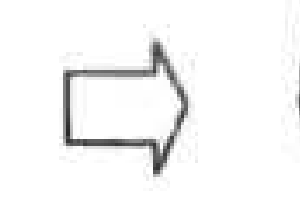

Impacto ambiental

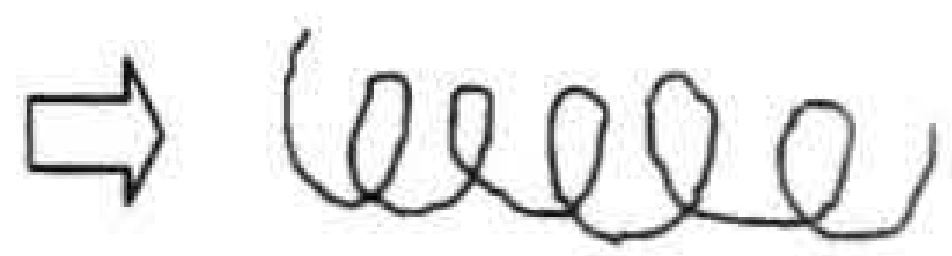

Resiliencia

Figura 6.8. Resiliencia. Elaboración propia

La resiliencia de un sistema natural lo hace más o menos vulnerable a las acciones antrópicas, como es el caso del diseño. El conocimiento de estas capacidades es muy importante para cuantificar el impacto ambiental de un producto.

\section{Conceptos}

"Amplitud de la tolerancia de un ambiente o ecosistema para asimilar perturbaciones sin deteriorarse definitivamente" (Ferrer-Véliz, 1978 citado por Sánchez, 1982:79).

\section{USOS O ACEPCIONES (ACLARACIONES)}

"Es una propiedad que permite que un sistema absorba y utilice el cambio (o incluso se beneficie de él)" (Holling, 1978 citado por Godoy, 2005:173).

"Capacidad de los sistemas ecológicos para absorber perturbaciones sin sufrir transformaciones cualitativas" (Libster citado por Godoy, 2005:173).

\section{SINÓNIMOS}

Adaptabilidad, flexibilidad, resistencia, tolerancia, adaptación, regeneración.

\section{ANTÓNIMOS}

Estático, rigido, inmutable.

\section{EJEMPLOS}

Los sistemas florestales que después de un extenso incendio, son capaces de absorber los impactos y regresar a su estado original.

\section{Bibliografía}

Godoy, Emiliano V. (2005), Diccionario de Ecologia, Argentina: Valletta Ediciones.

Sánchez Vicente; Beatriz Guiza (1982), Glosario de términos sobre medio ambiente, México: $\mathrm{El}$ Colegio de México.

Regenerative Development. Regenesis: More than green (www.regenesisgroup.com/articles Consultado el 25 de nov. de 2011).

\section{Sistema}

\section{DeFinición Diccionario RAE (DENOTACIÓN)}

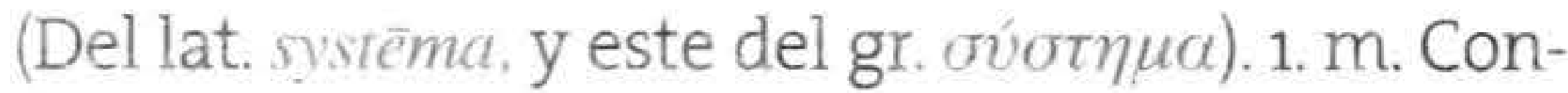
junto de reglas o principios sobre una materia racionalmente enlazados entre sí. 2. m. Conjunto de cosas que relacionadas entre sí ordenadamente contribuyen a determinado objeto. 3. m. Biol. Conjunto de órganos que intervienen en alguna de las principales funciones vegetativas. Sistema nervioso. 4. m. Ling. Conjunto estructurado de unidades relacionadas entre sí que se definen por 
oposición; por ejemplo, la lengua o los distintos componentes de la descripción lingüistica.

\section{DESARROLLO O CONSTRUCCIÓN DEL CONCEPTO}

La idea de sistema nos explica la organización de un conjunto de elementos que desempeñan un fin con base en sus interrelaciones en un determinado entorno o ambiente. Cada elemento del sistema cumple una función determinada y sin su participación el sistema se ve afectado. Por lo tanto, el sistema nos habla del conjunto de sus elementos como una totalidad integrada orgánica y funcionalmente. Un enfoque sistémico del ambiente natural y artificial hace alusión a una visión holística.

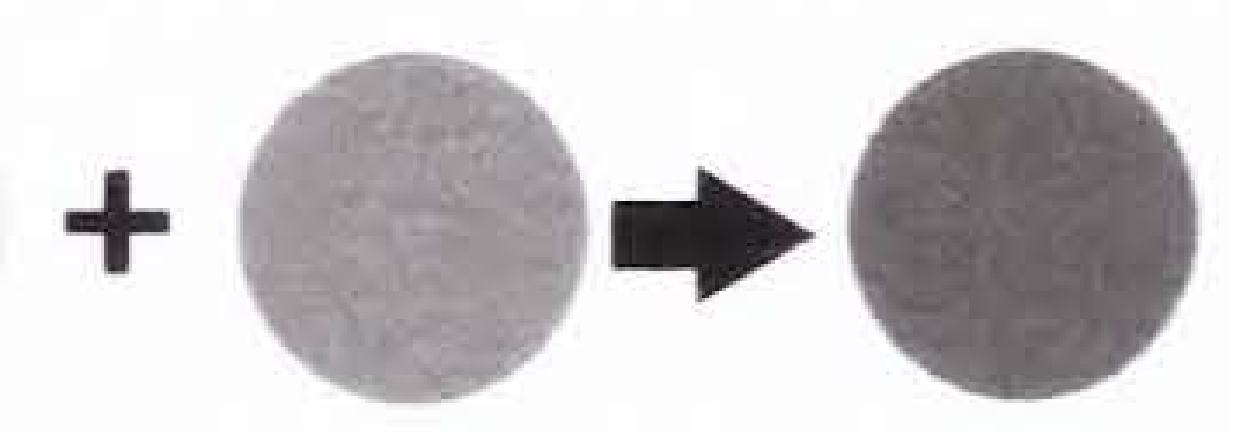

Figura 6.9. El resultado es más que la suma de las partes. Elaboración propia.

El diseñador se caracteriza por desarrollar una visión sistémica sobre los problemas que le toca resolver. Esto es relevante al desarrollar proyectos relacionados con la sustentabilidad, en virtud de los complejos conflictos planteados en este campo.

\section{Conceptos}

"Combinación de cosas o partes que forman un complejo o un todo unitario estrictamente".

"Conjunto coherente de elementos en interacción que pueden ser aislados del resto del universo con la ayuda de un criterio apropiado" (Allaby, 1984:367).

\section{USOS O ACEPCIONES (ACLARACIONES)}

Sistema de gestión ambiental

“Conjunto de medidas dirigidas al conocimiento, manejo y disminución de la incidencia negativa de las actividades de la organización sobre el medio ambiente. Forma parte del sistema general de gestión empresarial, por ello debe ser coherente con él y su diseño debe adaptarse a las características de la organización de que se trate" (Kent citado por Godoy, 2005:180).

- Sistema natural (naturaleza como sistema)

- Sistema de producción

- Sistema capitalista

- Diseño de sistemas

\section{SinóNIMOS}

Conjunto, organización, estructura, red.

\section{ANTónimos}

Unidad, individualidad, separación de elementos no relacionados.

\section{EJEMPLOS}

Sistema de comportamiento y tareas que se encuentran en el mundo de los insectos.

\section{Biblografía}

Allaby, Michael (1984). Diccionario del medio ambiente, España: Ediciones Pirámide. Godoy, Emiliano V. (2005), Diccionario de Ecología, Argentina: Valletta Ediciones.

Sánchez Vicente; Beatriz Guiza (1982), Glosario de términos sobre medio ambiente, México: El Colegio de México. 


\section{Sustentabilidad}

\section{Definición Diccionario RAE (DENOtACIÓN)}

Sustentabilidad. No existe definición en el Diccionario de la Real Academia Española de la Lengua.

Sostenible. Adj. Dicho de un proceso: que puede mantenerse por sí mismo, como lo hace, por ejemplo, un desarrollo económico sin ayuda exterior ni merma de los recursos existentes.

\section{DESARROLLO O CONSTRUCCIÓN DEL CONCEPTO}

La sustentabilidad es la caracteristica de un proceso de desarrollo que lo distingue por integrar la protección y el equilibrio ecológico, la equidad social, el crecimiento económico y el derecho a la cultura, de forma tal que las sociedades actuales satisfacen sus necesidades sin afectar las posibilidades de que en el futuro las próximas generaciones también lo hagan.

El diseño es una gran herramienta para impulsar el desarrollo económico y social, y por sus importantes vínculos con el medio natural, constituye un instrumento relevante para el desarrollo sustentable. El diseño, desde una perspectiva de la sustentabilidad, busca un equilibrio entre el medio artificial y el natural.

\section{Concepto}

“Capacidad de un ecosistema de mantener su estado igual, o bien equivalente, en el tiempo. Para lograrlo, se precisa el mantenimiento de ciertos parámetros por parte de la naturaleza a través de mecanismos de equilibrio dinámico" / Galván, 2007:240).

\section{USOS O ACEPCIONES (ACLARACIONES)}

Desarrollo sustentable

"El desarrollo es sustentable cuando satisface las necesidades de la presente generación sin comprometer la capacidad de las futuras generaciones para que satisfagan sus propias necesidades" (Gro Bruntland, 1987).

\section{Diseño sustentable}

"Es aquel diseño-proyecto que desde los requerimientos abarca los aspectos sociales, económicos, ecológicos y culturales del contexto específico al que va a atender en forma de un producto, servicio" (Margolin, 2005:33).

\section{SINÓNIMOS}

Sostenible, sustentable, económicamente viable, perdurable, infinito, eterno, fundamentado, justificado.

\section{Equilibrio y protección ecológica}

Derechos culturales

Crecimiento económico

Figura 6.10. Áreas de la sustentabilidad. Elaboración propia. 


\section{ANTÓNIMOS}

Insostenible, insustentable.

\section{EJEMPLOS}

Un ejemplo actual es la llamada pesca sustentable en donde la caza de peces (la producción) no daña la fuente de reproducción de peces (la base de recursos). Una pesca sustentable significa producir/cazar lo que necesitamos sin dañar los recursos de la producción.

\section{Bibliografía}

EDWARDS, Andrés (2005), The sustainability revolution: Portrait of a Paradigm Shift, New Society Publishers, EUA.

Galván Meraz, Francisco Javier (2007), Diccionario ambiental y asignaturas afines, Mundi Pensa Ediciones, México.

MARGolin, Víctor (2005), “Expansión o sustentabilidad: dos modelos de desarrollo", en Las politicas de lo artificial, Ed. Designio, México. Los estudios de la OECD en Desarrollo sustentable, disponibles en: http://www.oecd.org/about/o,33 47,en_2649_37425_1_1_1_1_37425, 00.html 